\title{
OPEN Microstructure of the fluid particles around the rigid body at the shear-thickening state toward understanding of the fluid mechanics
}

\author{
Ryota Jono $^{1 \bowtie}$, Syogo Tejima ${ }^{1}$ \& Jun-ichi Fujita ${ }^{2}$
}

We studied the shear-thickening behavior of systems containing rigid spherical bodies immersed in smaller particles using non-equilibrium molecular dynamics simulations. We generated shearthickening states through particle mass modulation of the systems. From the microstructures, i.e., two-dimensional pair distribution functions, we found anisotropic structures resulting from shear thickening, that are explained by the difference between the velocities of rigid bodies and fluid particles. The increasing viscosity in our system originated from collisions between fluid particles and rigid bodies. The lubrication forces defined in macroscale physics are then briefly discussed.

Shear-thickening fluids (or dilatant fluids) are non-Newtonian fluids ${ }^{1,2}$. It is important to elucidate the common principles of shear-thickening behavior because it widely appears from kitchens to factories in phenomena. Rigid spherical bodies immersed in a Newtonian fluid, such as silica particles in a polyethylene glycol solution, are wellknown systems exhibiting shear thickening that have been studied experimentally ${ }^{3,4}$. As a generalized system, rheological properties at the particle level have been well studied by simulation methods coupling equations of motion with Navier-Stokes equations and considering the Newtonian fluid as incompressible, as well as having a sufficiently low Reynolds number ${ }^{5-8}$. In these methods, forces acting on rigid bodies immersed in a Newtonian fluid are calculated as the sum of hydrodynamic forces characterizing interactions between rigid bodies and the fluid, non-hydrodynamic forces representing interactions between rigid bodies, and a Brownian term related to fluctuations owing to the system temperature. In recent studies, friction between solid particles has been found to play a role in determining the extent of the shear-thickening phenomenon ${ }^{8}$. The structure-property (viscosity) relation is studied by using the microstructure calculated by these methods ${ }^{5}$, and it has been experimentally verified $^{9,10}$. However, the origin and behavior of hydrodynamic forces are not fully clear from the viewpoint of particle interactions, although the analytical formula for one-body systems and the numerical formula for twobody systems exist ${ }^{11,12}$. Furthermore, the microstructure of fluid has not been discussed so far because the fluid is treated as continuum model in these macroscale simulation methods.

In a previous study, we performed non-equilibrium molecular dynamics (NEMD) simulations to analyze the shear-thickening state of a polymer in an aqueous solution ${ }^{13}$. This type of NEMD simulation is useful for studying fluid particles as explicit atoms or molecules to reveal the characteristic properties of the hydrodynamic forces. In this work, we used NEMD simulations to reproduce shear thickening and analyzed the origin of the viscosity changes from the viewpoint of particles to connect the molecular-scale information with macroscale physics. The aim of this study is to reproduce the macroscale physics using molecular-level simulations that considers only the interaction between particles and does not explicitly consider the lubrication force and the friction forces that should be defined in macroscale physics. We report that the origin of the shear-thickening phenomenon is not only the collisions between rigid bodies, which are calculated by macroscale simulations, but also those between rigid bodies and fluid particles.

${ }^{1}$ Research Organization for Information Science and Technology, 7F, Sumitomo-Hamamatsucho Building, 1-18-16, Hamamatsucho, Minato-ku, Tokyo 105-0013, Japan. ${ }^{2}$ Institute of Applied Physics, Graduate School of Pure and Applied Science, University of Tsukuba, Tsukuba, Ibaraki 1-1-1 Ten-nodai, Tsukuba, Ibaraki 305-8573, Japan. ${ }^{\square}$ email: jono@rist.or.jp 


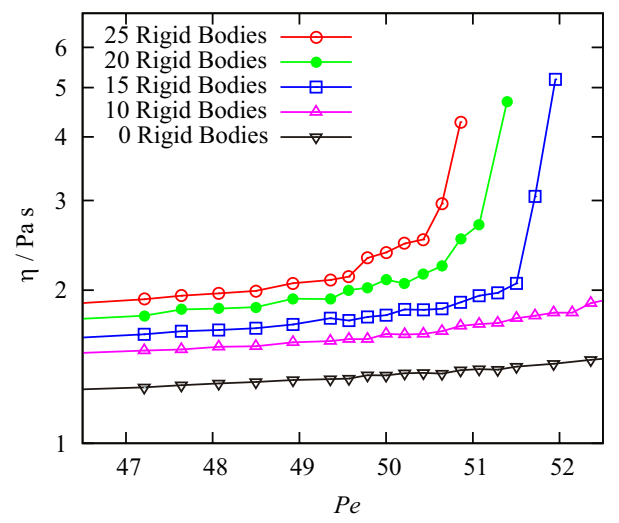

Figure 1. Rheology plot of the calculated systems composed of $0,10,15,20$, and 25 rigid bodies immersed in the particles.

\section{Results}

Rheology plot. Figure 1 shows the viscosity as a function of the Péclet number, that is, the rheology plot. All curves except for the reference system (i.e., 0 rigid bodies) exhibit shear thickening. The critical shear rate for shear thickening decreases when the volume fraction (or concentration) of the rigid body increases, which is in good agreement with the experimental results ${ }^{2,3}$. To elucidate the origin of shear thickening, we focused on a system composed of 20 rigid bodies immersed in 2600 fluid particles. In the following discussion, we divide the related rheology plot into two regions, namely, a Newtonian-fluid region for $P e<50$ and a shear-thickening-fluid region for $P e>50$.

Radial distribution function. Figure 2a shows the radial distribution functions of the fluid particles $g_{\mathrm{pp}}(r)$. As $P e$ increases, the height and width of the first solvation shell in $g_{\mathrm{pp}}(r)$ become higher and narrower, respectively, in the Newtonian-fluid region $(P e<50)$. This indicates that the shear flow limits the distance between fluid particles. Increasing $P e$ beyond 50 (i.e., in the shear-thickening-fluid region) causes the height and width of the first solvation shell in $g_{\mathrm{pp}}(r)$ to be lower and broader, respectively. This indicates that the solvation structure collapses when the shear thickening starts. However, changes in $g_{\mathrm{pp}}(r)$ for both the Newtonian-fluid and shearthickening-fluid regions remain small. Figure $2 \mathrm{~b}$ shows the radial distribution functions between the centers of mass $(\mathrm{COM})$ of the rigid bodies and the fluid particles, $g_{\mathrm{Rp}}(r)$, which are related to hydrodynamic forces. The behavior of the peak related to the first solvation shell is similar to that of $g_{\mathrm{pp}}(r)$. However, there is a remarkable peak at $r=5.6 \AA$, corresponding to fluid particles located in the hollow sites of pentagons inherent in the dodecahedral structure of rigid bodies, as shown in the inset of Fig. $2 \mathrm{~b}$. The peak at $r=5.6 \AA$ increases with increasing shear rate. Figure $2 \mathrm{c}$ shows the pair distribution functions between the COMs of the rigid bodies, $g_{\mathrm{RR}}(r)$. As the Péclet number increases, the peak heights and widths of $g_{\mathrm{RR}}(r)$ become broader and lower, respectively, in both the Newtonian-fluid and the shear-thickening-fluid regions, but the degree of broadening in the shear-thickening-fluid region is larger than that in the Newtonian-fluid region. These results indicate that shear induces collisions between rigid bodies and fluid particles, and between rigid bodies. However, the relationship between the aforementioned collisions and shear thickening remains unclear.

Microstructure. The system microstructures were further investigated using two-dimensional pair distribution functions, $h(x, y)$, as shown in Fig. 3. For the pair distribution function between fluid particles, $h_{\mathrm{pp}}(x, y)$, we found isotropic structures among $P e=45,49$, and 51.5, meaning that the shear rate had only a slight effect. It is agreement with the previous simulation results ${ }^{14}$. There were remarkable changes in the two-dimensional pair distribution functions between rigid bodies and fluid particles, $h_{\mathrm{Rp}}(x, y)$. With increasing Péclet number, the isotropic microstructure at $P e=45$ became anisotropic at $P e=51.5$ (Fig. 3f). The velocities of fluid particles in the $y>0, x<0$ region were higher than those of rigid bodies, assuming that the velocities of rigid bodies were well represented by the velocity of the COMs. Therefore, fluid particles at $y>0$ and $x<0$ caught up with and crashed into rigid bodies. The jamming effect ${ }^{8,15}$ appeared because the motion of particles is restricted at high Péclet numbers and cannot leave the layer formed by the shear flow. Rigid bodies cannot catch up with fluid particles at $y>0$ and $x>0$. Therefore, a sparse distribution is formed. These competitions between fluid particles and rigid bodies are also held in the $y<0$ region, so an anisotropic but symmetric microstructure is formed at high Péclet numbers. The origin of these behaviors is the interaction between fluid particles and rigid bodies. Therefore, interactions such as lubrication forces defined in macroscale physics may be important factors in the origin of shear thickening.

In addition, the two-dimensional pair distribution functions between rigid bodies $\left(h_{\mathrm{RR}}(x, y)\right)$ showed the presence of anisotropic structures, even in the Newtonian-fluid domain, with well-aligned rigid bodies forming layers owing to the shear flow at high Péclet numbers. A rigid body can approach another rigid body within a distance of $1 \mathrm{~nm}$ if they are located in the same layer formed by the shear flow (i.e., the $y$-position is the same in the velocity gradient). However, rigid bodies cannot migrate between the layers formed by shear flow. Therefore, 

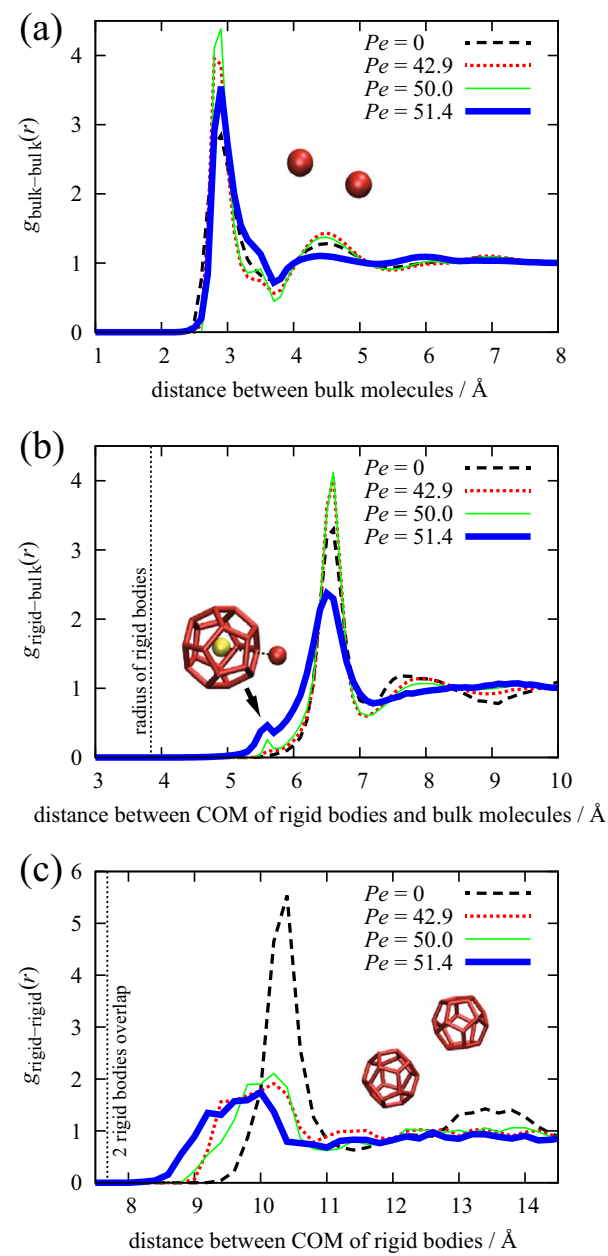

Figure 2. Radial distribution functions of 20 rigid bodies immersed in 2600 particles between (a) particle and particle, (b) COM (yellow particle shown in the inset) of the rigid body and particles, and (c) COM of the rigid bodies and COM of the rigid bodies for each $P e=42.9$ (at Newtonian region), 50 (at critical Pe), and 51.4 (at shear-thickening region).

the distance between two rigid bodies belonging to two different layers formed by shear flow is greater than $2 \mathrm{~nm}$, meaning that collisions between them are less likely to occur. Therefore, the main influence on the viscosity of the system is the collisions between fluid particles and rigid bodies. It should be noted that collisions between rigid bodies correspond to the results of the macroscale simulations.

From molecular-scale physics to macroscale physics. To deepen our understanding of the lubrication forces of fluid particles, we performed additional simulations. We built a system composed of two rigid bodies immersed in fluid particles. They were separated by a distance of $2 \mathrm{~nm}$ and aligned along the velocity gradient direction (i.e., the $y$-direction). We compared the velocity distributions obtained for this system with those corresponding to a system with only fluid particles ( 0 rigid bodies). In the latter case, the velocity distribution in the shear flow direction depicts a gradient, as generally expected for Couette flow generated by the SLLOD method Fig. 4a. In our system, where the rigid bodies were placed in the flow, the velocity distribution of fluid particles located between the two rigid bodies was modified and exhibited fluid-particle-averaged velocities in the shear flow direction of approximately zero in the range of $y=-10$ to $y=+10$, as shown in Fig. 4b. This effect, called lubrication forces in macroscale physics, appeared in the molecular-scale simulations. The jamming effect seems to occur at $(x, y)=(-5,10)$ or $(5,-10)$ in Fig. $4 \mathrm{~b}$ because the velocities at those points are almost zero. Further investigations of this effect on the molecular scale will be useful for better understanding the principles of macroscale physics.

In conclusion, we performed non-equilibrium molecular dynamics simulations and observed shear thickening by modifying the mass of the system to reproduce high-Péclet-number conditions. Our molecular-scale analysis showed that shear thickening originates from collisions between fluid particles and rigid bodies, that is, collisions between entities of different sizes. Based on these results, the rheology plot shown in Fig. 1, which shows the concentration dependency of the viscosity, can be explained by the increase of the collision frequency between fluid particles and rigid bodies with increasing the concentration of the rigid bodies. 
(a)

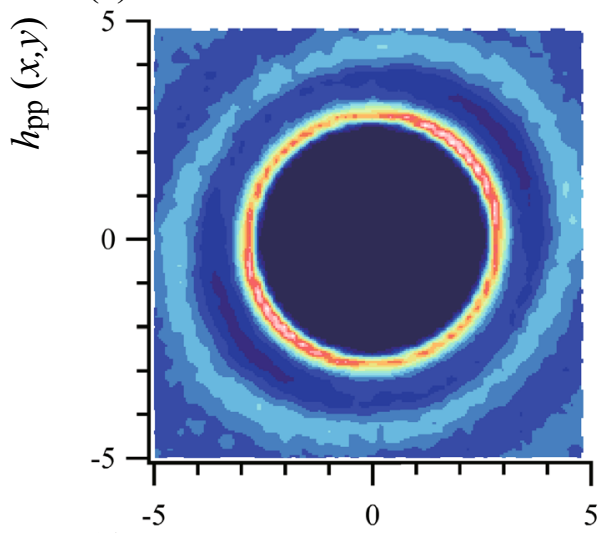

(d)

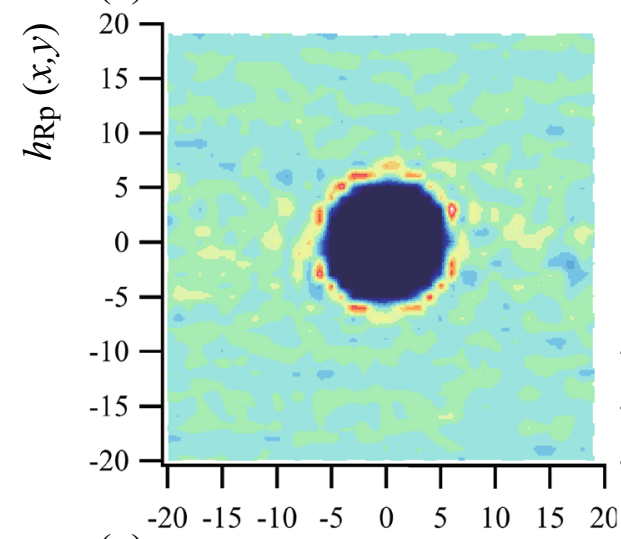

(g)

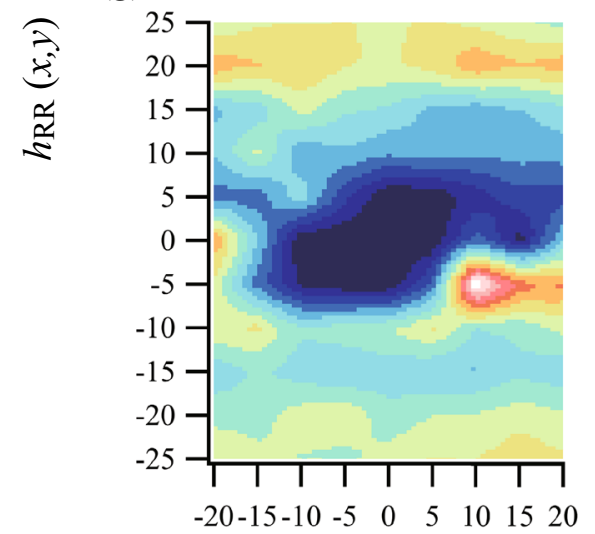

(b)

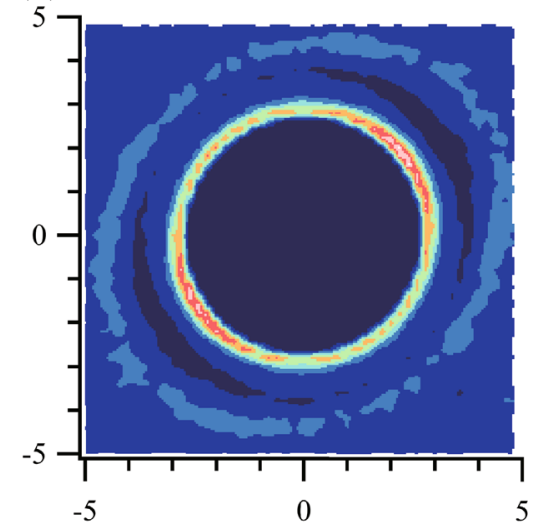

(e)

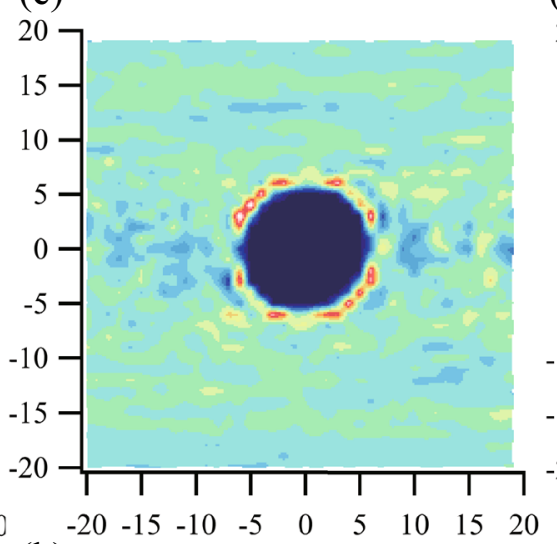

(h)

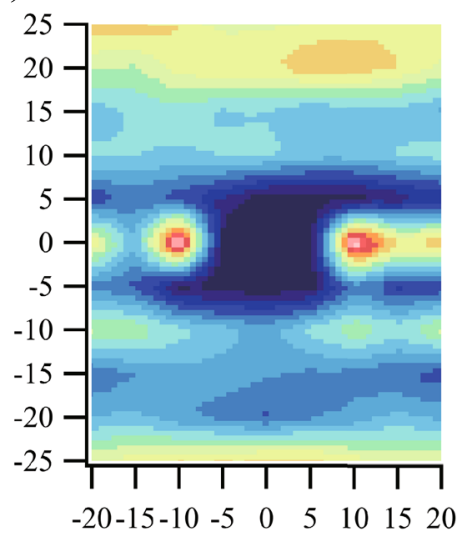

(c) $\quad P e=51.4$

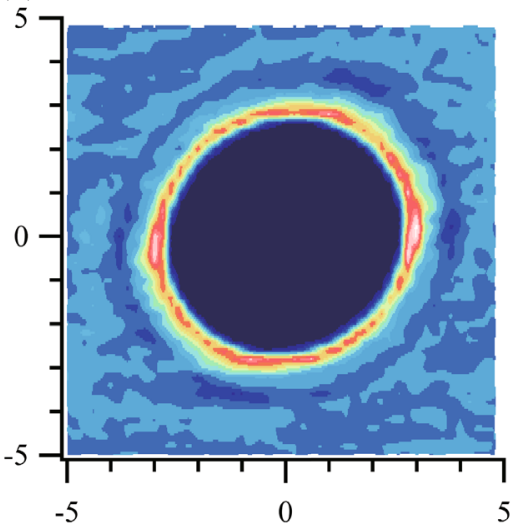

(f)

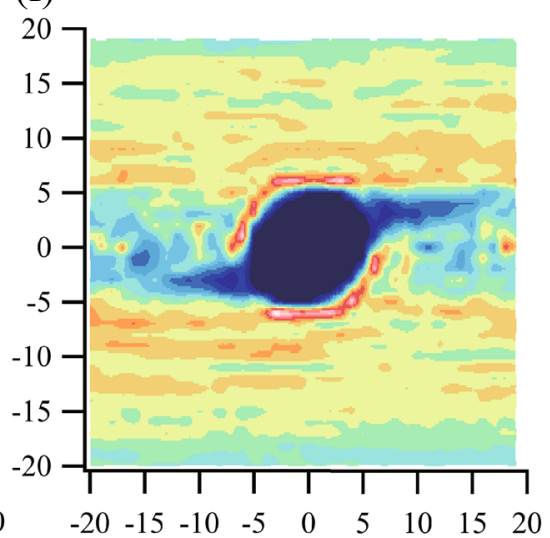

(i)

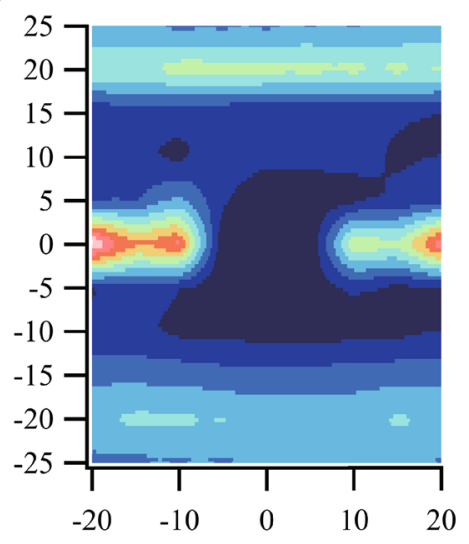

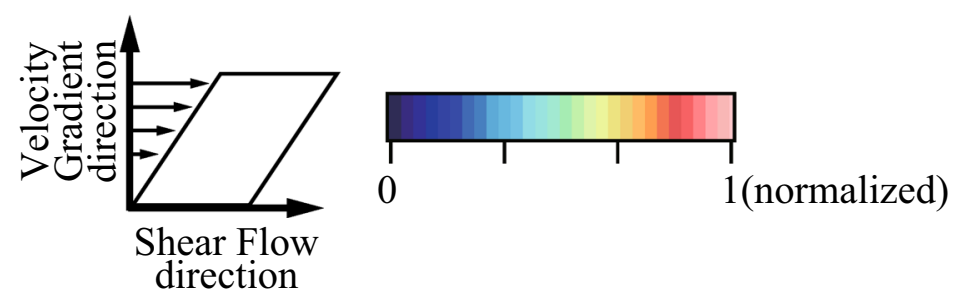

Figure 3. Pair distribution functions of the 20 rigid bodies immersed in 2600 particles (a)-(c) between particle and particle, $(\mathbf{d})-(\mathbf{f})$ between COM of the rigid body and particles, and ( $\mathbf{g})-(\mathbf{i})$ between COM of the rigid bodies and $\mathrm{COM}$ of the rigid bodies for each $P e=42.9$ (at Newtonian region), 50 (at critical $P e$ ), and 51.4 (shearthickening region). 
(a)

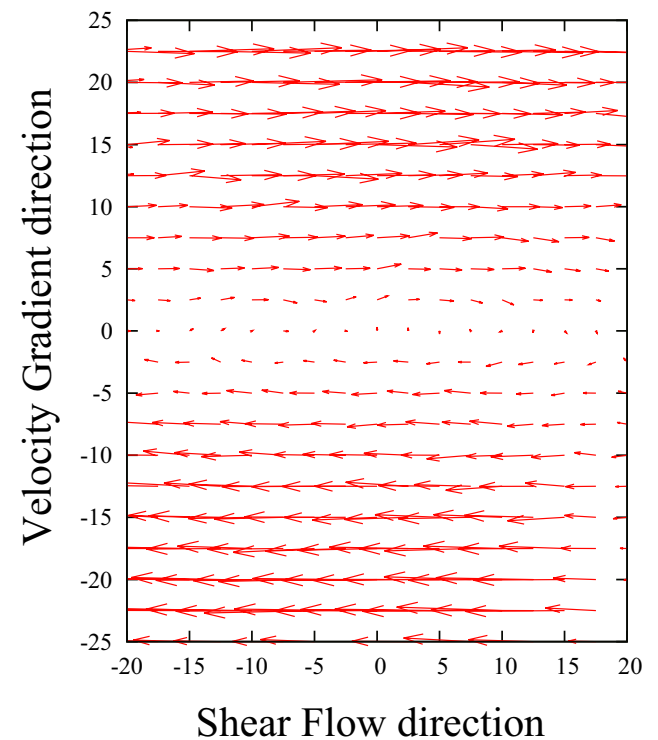

(b)

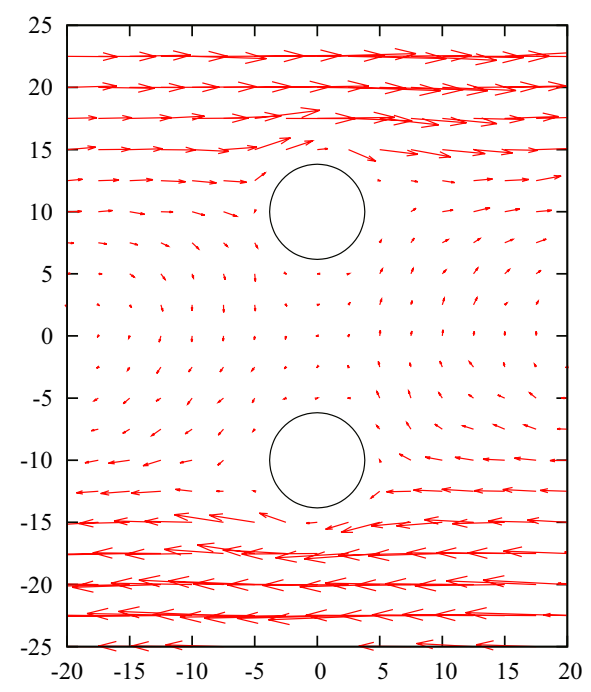

Figure 4. The velocity distribution of the particles (a) in the shear flow direction and (b) between two rigid bodies, which are represented by circles.

\section{Methods}

The systems used comprised 3000 same-sized particles. Each rigid body contained 20 smaller particles placed at the 20 vertices of a dodecahedron. Then, the positions of the particles in the dodecahedron were optimized so that the maximum force did not exceed $2 \times 10^{-3} \mathrm{kcal} \cdot \mathrm{mol}^{-1} \AA^{-2}$ to eliminate any influence on the viscosity calculations. The numbers of rigid bodies considered in this study were $0,10,15,20$, and 25 . The Stillinger-Weber force field ${ }^{16}$ was used to describe the interactions between particles. The viscosity $(\eta)$ at a given shear rate $(\dot{\gamma})$ is expressed as $\eta(\dot{\gamma})=-\left\langle P_{x y}\right\rangle / \dot{\gamma}$, where $\left\langle P_{x y}\right\rangle$ is the ensemble average of the pressure tensor element $P_{x y}$. The standard deviation of $\eta$ depends on shear rate $\dot{\gamma}$ because that of $P_{x y}$ does not depend on the shear rate. The standard deviation of $\eta$ is sufficiently small for $\dot{\gamma}$ values larger than $10^{8} \mathrm{~s}^{-1}$. Therefore, it is important to find the conditions under which shear thickening occurs at $\dot{\gamma}=10^{8} \mathrm{~s}^{-1}$. It has been reported that shear thickening occurs when the Péclet number, $P e=\dot{\gamma} a^{2} / D$, is approximately $100^{17,18}$, where $a$ is the representative length and $D$ is the diffusion coefficient of the system. Based on this rule of thumb, we multiplied the mass of the particles by $10^{6}$ because the diffusion coefficient should be modified from $\sim 10^{-9} \mathrm{~m}^{2} \mathrm{~s}^{-1}$ to $\sim 10^{-11} \mathrm{~m}^{2} \mathrm{~s}^{-1}$ considering the diameter of regular dodecahedrons $(\sim 1 \mathrm{~nm})$ as the representative length, and the applied shear rate was $\sim 10^{8} \mathrm{~s}^{-1}$. The weight of the mass modulated dodecahedral structure is similar to that of the silica particle with tens of nanometers of diameter if $\rho=2.2 \mathrm{gcm}^{-3}$ is used. Thus, the calculated diffusion coefficient of the system was noted to be $5 \times 10^{-12} \mathrm{~m}^{2} \mathrm{~s}^{-1}$, which is similar to that of polyethylene glycol $\left(D \approx 10^{-11} \mathrm{~m}^{2} \mathrm{~s}^{-1}\right)$. The volume for all systems was adjusted in the $N p T$ ensemble, resulting in mass densities of $1.00,0.99,0.98,0.97$, and $0.97 \mathrm{~kg} \mathrm{~mm}^{-3}$ for the $0,10,15,20$ and 25 rigid-body systems, respectively. The SLLOD method ${ }^{19}$ under the NVT simulation was used to apply shear. The Nosé-Hoover thermostat was used to control the temperature of the systems at $300 \mathrm{~K}$. All calculations were performed using LAMMPS ${ }^{20}$. Production runs of $100 \mathrm{~ns}$ were performed with a time step of 10 fs. To ensure a steady state, only the last 99 ns of the production run was used for sampling $P_{x y}$ and the molecular structure, with sampling rates of $0.1 \mathrm{ps}$ and $100 \mathrm{ps}$, respectively.

Received: 2 September 2021; Accepted: 8 December 2021

Published online: 17 December 2021

\section{References}

1. Brown, E. \& Jaeger, H. M. Shear thickening in concentrated suspensions: Phenomenology, mechanisms and relations to jamming. Rep. Prog. Phys. 77, 046602 (2014).

2. Hasanzadeh, M. \& Mottaghitalab, V. The role of shear-thickening fluids (STFs) in ballistic and stab-resistance improvement of flexible armor. J. Mater. Eng. Perform. 23, 1182 (2014).

3. Kamibayashi, M., Ogura, H. \& Otsubo, Y. Shear-thickening flow of nanoparticle suspensions flocculated by polymer bridging. J. Colloid. Inter. Sci. 321, 294 (2008).

4. Lee, J., Jiang, Z., Wang, J., Sandy, A. R. \& Narayanan, S. Unraveling the role of order-to-disorder transition in shear thickening suspensions. Phys. Rev. Lett. 120, 028002 (2018).

5. Brady, J. F. \& Bossis, G. Stokesian dynamics. Ann. Rev. Fluid Mech. 20, 111 (1988).

6. Chen, K., Wang, Y., Xuan, S. \& Gong, X. A hybrid molecular dynamics study on the non-Newtonian rheological behaviors of shear thickening fluid. J. Colloid Interf. Sci. 497, 378 (2017).

7. Nakayama, Y. \& Yamamoto, R. Simulation method to resolve hydrodynamic interactions in colloidal dispersions. Phys. Rev. E 71, 036707 (2005). 
8. Seto, R., Mari, R., Morris, J. F. \& Denn, M. M. Discontinuous shear thickening of frictional hard-sphere suspensions. Phys. Rev Lett. 111, 218301 (2013).

9. Parsi, F. \& Gadala-Maria, F. Fore-and-aft asymmetry in a concentrated suspension of solid spheres. J. Rheol. 31, 825 (1987)

10. Cheng, X., McCoy, J. H., Israelachvili, J. N. \& Cohen, I. Imaging the microscopic structure of shear thinning and thickening colloidal suspensions. Science 333, 1276 (2011).

11. Brenner, H. \& O’Neill, M. E. On the stokes resistance of multiparticle systems in a linear shear field. Chem. Eng. Sci. 27, 1421 (1972).

12. Mazur, P. \& Saarloos, W. V. Many-sphere hydrodynamic interactions and mobilities in a suspension. Physica 115A, 21 (1982).

13. Bonnaud, P. A., Ushiyama, H., Tejima, S. \& Fujita, J.-I. Neat and aqueous polyelectrolytes under a steady-shear flow. J. Phys. Chem. $B$ 125, 6930 (2021).

14. Hanley, H. J. M., Rainwater, J. C. \& Hess, S. Shear-induced angular dependence of the liquid pair correlation function. Phys. Rev. A 36, 1795 (1987).

15. Fall, A., Bertrand, N. H. F., Ovarlez, G. \& Bonn, D. Shear thickening of cornstarch suspensions as a reentrant jamming transition. Phys. Rev. Lett. 100, 018301 (2008).

16. Chan, H. et al. Machine learning coarse grained models for water. Nat. Commun. 10, 379 (2019).

17. Nakamura, H., Ishii, M. \& Makino, S. Dominant model of shear thickening behavior of concentrated suspensions of monodispersed colloidal particles. J. Soc. Powder Technol. Jpn. 56, 438 (2019).

18. Bender, J. \& Wagner, N. J. Reversible shear thickening in monodisperse and bidisperse colloidal dispersions. J. Rheology 40, 899 (1996).

19. Evans, D. J. \& Morriss, G. P. Nonlinear-response theory for steady planar couette flow. Phys. Rev. A 30, 1528 (1984).

20. Plimpton, S. Fast parallel algorithms for short-range molecular dynamics. J. Comput. Phys. 117, 1 (1995).

\section{Acknowledgements}

This work was financially supported by the Innovative Science and Technology Initiative for Security Grant Number JPJ004596, ATLA, Japan, and the computational resources provided by the Multidisciplinary Cooperative Research Program in the Center for Computational Sciences, University of Tsukuba.

\section{Author contributions}

R.J., S.T. and J-i.F. developed the basic concept. R.J. performed the simulation.

\section{Competing interests}

The authors declare no competing interests.

\section{Additional information}

Correspondence and requests for materials should be addressed to R.J.

Reprints and permissions information is available at www.nature.com/reprints.

Publisher's note Springer Nature remains neutral with regard to jurisdictional claims in published maps and institutional affiliations.

(c) (i) Open Access This article is licensed under a Creative Commons Attribution 4.0 International License, which permits use, sharing, adaptation, distribution and reproduction in any medium or format, as long as you give appropriate credit to the original author(s) and the source, provide a link to the Creative Commons licence, and indicate if changes were made. The images or other third party material in this article are included in the article's Creative Commons licence, unless indicated otherwise in a credit line to the material. If material is not included in the article's Creative Commons licence and your intended use is not permitted by statutory regulation or exceeds the permitted use, you will need to obtain permission directly from the copyright holder. To view a copy of this licence, visit http://creativecommons.org/licenses/by/4.0/.

(C) The Author(s) 2021 